\title{
Novel method for detecting micro-organisms in blood cultures
}

\author{
D SAWHNEY,* S HINDER, $\dagger$ D SWAINE,* E Y BRIDSON* \\ From the Departments of *Oxoid Limited, Basingstoke, and the $†$ Basingstoke District Hospital, Basingstoke
}

SUMMARY A method for detecting the growth of micro-organisms in blood culture by a visual signal is described. The system utilises a single blood culture medium that has been specifically formulated to support growth of aerobic, anaerobic, and microaerophilic micro-organisms. The system is based on the principle that when micro-organisms grow in the medium in a sealed bottle their metabolic products create positive pressure. This positive pressedre displaces the infected blood and broth into an upper chamber, which acts as a visual signal of microbial activity. All the test micro-organisms, when inoculated at $<20$ colony forming units into simulated human blood cultures. gave a positive signal.

In the past few years several attempts have been made to improve the early recognition of microbial growth in blood cultures. Recent examples of systems developed to achieve this are: the Bactec System (Bactec Johnston Laboratories, Cockeysville, Maryland) where microbial growth is indicated by the release of radioactively labelled carbon dioxide from growth substrates; the Septi-Check BCB System (Roche Diagnostics), which consists of a routine blood culture bottle on to which can be attached a cylinder that contains an agar coated slide. The slide media are inoculated by tipping the bottle so that the blood and broth flows over the slide. Microbial growth is indicated by the presence of colonies growing on the agar surface; and the Malthus System, which indicates microbial growth by measuring a change in the electrical impedence.

Each of these systems has advantages and disadvantages, and after studying these products it was decided to develop a new system with the following criteria:

1 Signal the presence of metabolising microorganisms.

2 No radioactive isotopes.

3 No hardware support or electrical signalling.

4 Suitable for large or small scale use.

5 Recover very small numbers of micro-organisms.

6 Rapid growth of micro-organisms.

7 A single medium suitable for aerobic, anaerobic, and microaerophilic micro-organisms.

8 Easy to sample.

9 No subculture contamination.

10 User friendly - that is safe, easy, and reliable.

The criteria described above were achieved with Accepted for publication 14 May 1986 this new blood culture system. The purpose of this paper is to describe the principle of operation, the design of the system, and report the results obtained with simulated human blood cultures.

\section{THE PRINCIPLE}

Microbial growth in a medium that has been specifically formulated will create a positive pressure in a sealed container. The formulation of the medium ensures that this metabolic effect is produced by aerobic, anaerobic, and microaerophilic micro-organisms. The detection of positive pressure is by means of a secondary chamber (signal device) that is connected to the bottle after the blood sample has been added. A positive pressure in the bottle displaces a sample of the infected blood and broth into the signal device and hence indicates microbial activity.

\section{Material and methods}

\section{COMPONENTS OF THE SYSTEM}

1 A sealed bottle containing $36.5 \mathrm{ml}$ of special broth medium under vacuum.

2 A sterile growth signal device which is vented through a $0.22 \mu \mathrm{m}$ hydrophobic filter (figure).

(The system has been changed to a bottle containing $80 \mathrm{ml}$ of broth, capable of taking up to $10 \mathrm{ml}$ of blood. The signal device has also been changed in design but not in function.)

\section{MEDIUM FORMULATION}

The medium was specifically formulated to support the growth of aerobic, anaerobic, and microaerophilic micro-organisms, designed so that during growth of micro-organisms the metabolic effect was 


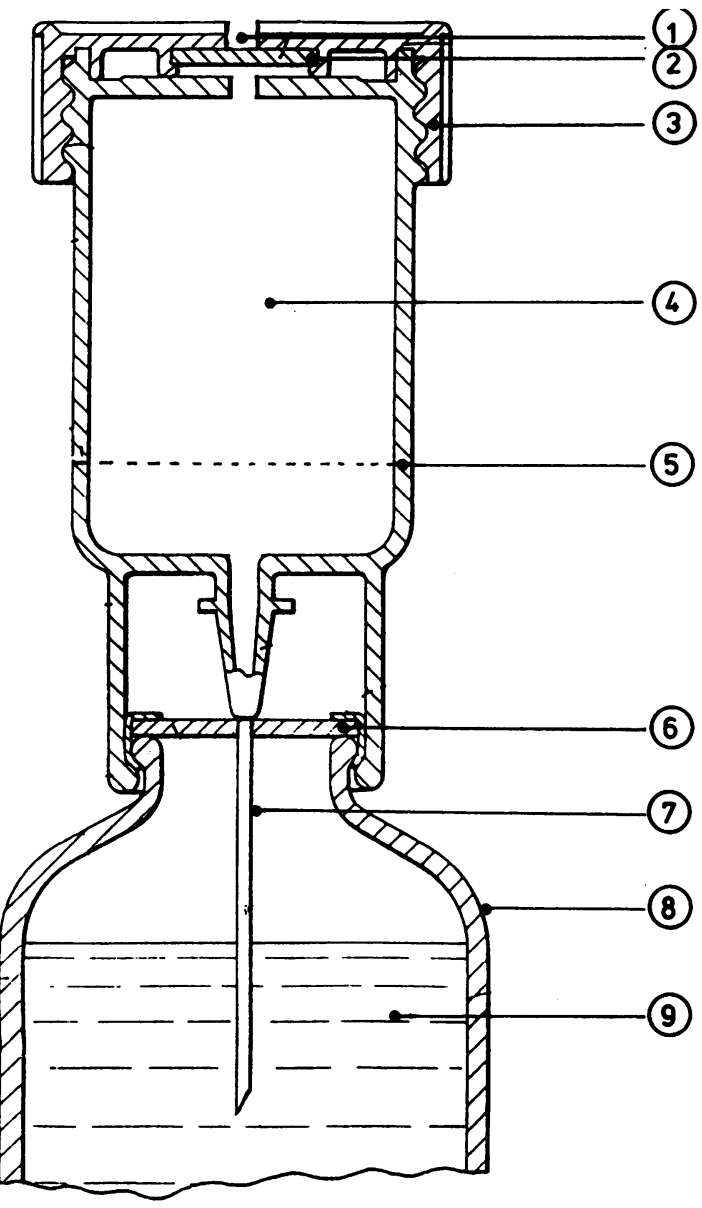

Figure Blood culture system $\quad 1=$ vent; $2=0.22 \mu \mathrm{m}$ hydrophobic filter; $3=$ signal device cap; $4=$ signal device chamber-total capacity $15 \mathrm{ml} ; 5$ = opaque region to mask the blood and broth displacement due to temperature increase-displacement above this mark with positive blood samples; 6 = blood culture bottle rubber diaphragm;

$7=50 \mathrm{~mm}$ hyperdermic needle; $8=$ blood culture bottle;

$9=$ blood culture medium.

to create a positive pressure and in the initial 24 hours' incubation to give $2 \cdot 5-5.0 \%$ carbon dioxide in the bottle head space (table 1).

\section{THE OPERATION OF THE SYSTEM}

1 Blood sample $(5 \mathrm{ml})$ is added to each bottle of broth-the system is also functional for smaller blood volumes. The contents of the bottle are under vacuum and this ensures that no positive pressure aerosols are produced on removal of the syringe needle.

2 On return to the laboratory the signal device is connected to the bottle by inserting its needle through
Table 1 Formulation suitable for supporting growth of aerobic, anaerobic, and microaerophilic micro-organisms (European Patent 0124193 Al)

\begin{tabular}{ll}
\hline Tryptone soya broth & $10 \mathrm{~g} / \mathrm{l}$ \\
Gelatin peptone & 10 \\
Yeast extract & 5 \\
Meat extract & 5 \\
Sodium chloride & 8 \\
Potassium nitrate & 2 \\
Glucose & 1 \\
L-arginine & 1 \\
Sodium pyruvate & 1 \\
Gelatin & 1 \\
Sodium thioglycollate & 0.5 \\
Cysteine hydrochloride & 0.4 \\
Sodium bicarbonate & 0.4 \\
Phosphate buffer & 0.3 \\
Sodium polyanethol sulphonate & 0.3 \\
Dithiothreitol & 0.2 \\
Adenine sulphate & 0.01 \\
Sodium succinate & 0.01 \\
Ammonium chloride & 0.008 \\
Magnesium sulphate & 0.008 \\
Menadione & 0.005 \\
\hline
\end{tabular}

Table 2 Time of positive signal obtained from number of colony forming units inoculated

\begin{tabular}{lll}
\hline & $\begin{array}{l}\text { Inoculum } \\
\text { (colony } \\
\text { forming } \\
\text { units) }\end{array}$ & $\begin{array}{l}\text { Time of } \\
\text { positive } \\
\text { signal } \\
\text { (hours) }\end{array}$ \\
\hline Organism & 13 & 72 \\
\hline Peptostreptococcus sp & 8 & 44 \\
*Bacteroides sp & 2 & 24 \\
*Pseudomonas aeruginosa & 1 & 24 \\
*Staphylococcus aureus & 4 & 20 \\
*Escherichia coli & 8 & 30 \\
*Streptococcus pyogenes & 3 & 20 \\
*Streptococcus pneumoniae & 10 & 17 \\
Streptococcus faecalis & 6 & 20 \\
*Klebsiella pneumoniae & 12 & 54 \\
Haemophilus influenzae & &
\end{tabular}

*These organisms were originally isolated from positive blood cultures at Basingstoke District Hospital. This part of study was carried out at the hospital. All the positive blood cultures when subcultured from the signal device had viable organisms.

Table 3 Time of positive signal obtained from number of colony forming units inoculated

\begin{tabular}{lll}
\hline & $\begin{array}{l}\text { Inoculum } \\
\text { (colony } \\
\text { forming } \\
\text { units) }\end{array}$ & $\begin{array}{l}\text { Time of } \\
\text { positive } \\
\text { signal } \\
\text { (hours) }\end{array}$ \\
\hline Organism & 17 & 64 \\
\hline Neisseria meningitidis & 3 & 24 \\
Listeria monocytogenes & 9 & 78 \\
Mycoplasma hominis & 7 & 24 \\
Gardnerella vaginalis & 4 & 24 \\
Yersinia enterocolitica & 4 & 72 \\
Legionella pneumophila & 4 &
\end{tabular}

This part of the study was carried out in the Oxoid Laboratories using human blood. All the positive blood cultures, when subcultured from the signal device, had viable organisms. 
the rubber diaphragm. The signal device chamber has a total capacity of $15 \mathrm{ml}$ and is attached to a $50 \mathrm{~mm}$ hyperdermic needle. The signal device cap is vented to atmosphere through a $0.22 \mu \mathrm{m}$ hydrophobic filter. During the insertion of the signal device needle through the rubber diaphragm the tip of the needle is retained in the bottle head space for five seconds to allow equilibrium of air pressure to be established. This prevents the inspiration of air into the reduced broth. After equilibrium the needle is pushed into the blood and broth.

3 The system is then transferred to a $35^{\circ} \mathrm{C}$ incubator. Due to an increase of temperature $\left(18^{\circ} \mathrm{C}\right.$ to $35^{\circ} \mathrm{C}$ ) an initial expansion of head space air increases the pressure and displaces $2.3 \mathrm{ml}$ of blood and broth into the signal device chamber. This displacement indicates that the system is not leaking and in the final design will be masked by an opaque region. A positive signal is recognised when the infected blood and broth is above the opaque region. The infected blood and broth continues to be displaced into the signal device chamber until the needle tip is free from the infected blood and broth in the bottle. At this stage all excess pressure is vented through the $0.22 \mu \mathrm{m}$ hydrophobic filter sealed in the signal device cap. The bottles that show a positive signal are removed from the incubator.

4 A sample of the infected blood and broth is taken from the signal device chamber by unscrewing the vented cap. The venting of the system ensures that the signal device chamber is always at ambient pressure. 5 The negative bottles are incubated at $35^{\circ} \mathrm{C}$ up to

Table 4 Gas analysis of blood culture bottles head space after addition of freshly collected human blood

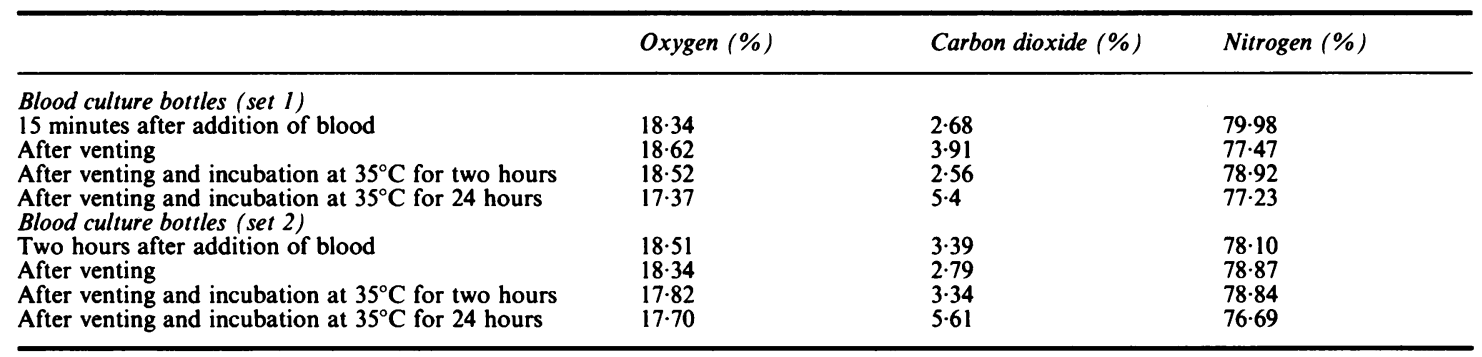

Table 5 Micro-organisms inoculated into blood culture system $(<20$ cfu) and generating positive growth signal (figures in parentheses show No of separate strains tested)

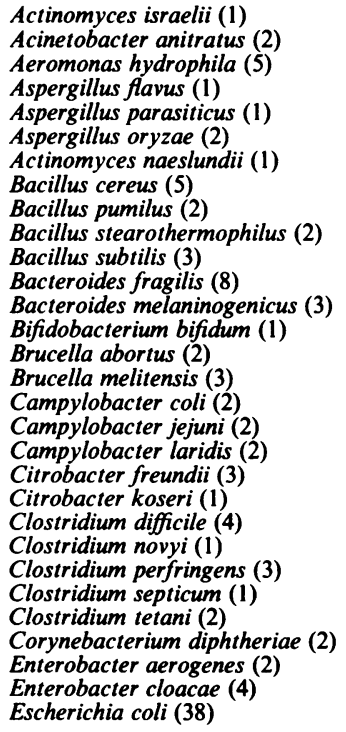

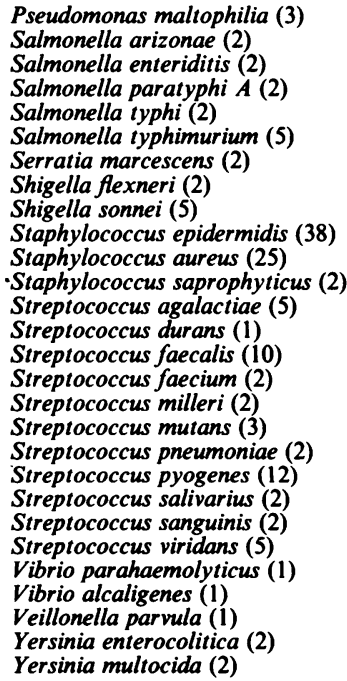


seven days. Each day of incubation the bottles are examined for growth and gently agitated (morning and late afternoon) to resuspend red blood cells.

6 The positive and negative bottles are sterilised by autoclaving before disposal.

\section{SIMULATED HUMAN BLOOD CULTURES}

The blood culture system was challenged with a panel of test organisms (tables 2 and 3).

1 Serial dilutions of an overnight broth culture of each test organism were prepared.

2 Drops $(50 \mu \mathrm{l})$ of each dilution were inoculated on to blood or chocolate agar plates and incubated at $35^{\circ} \mathrm{C}$ in the appropriate atmosphere requirement for each organism.

Table 6 Micro-organisms isolated from blood culture trials carried out in various hospitals

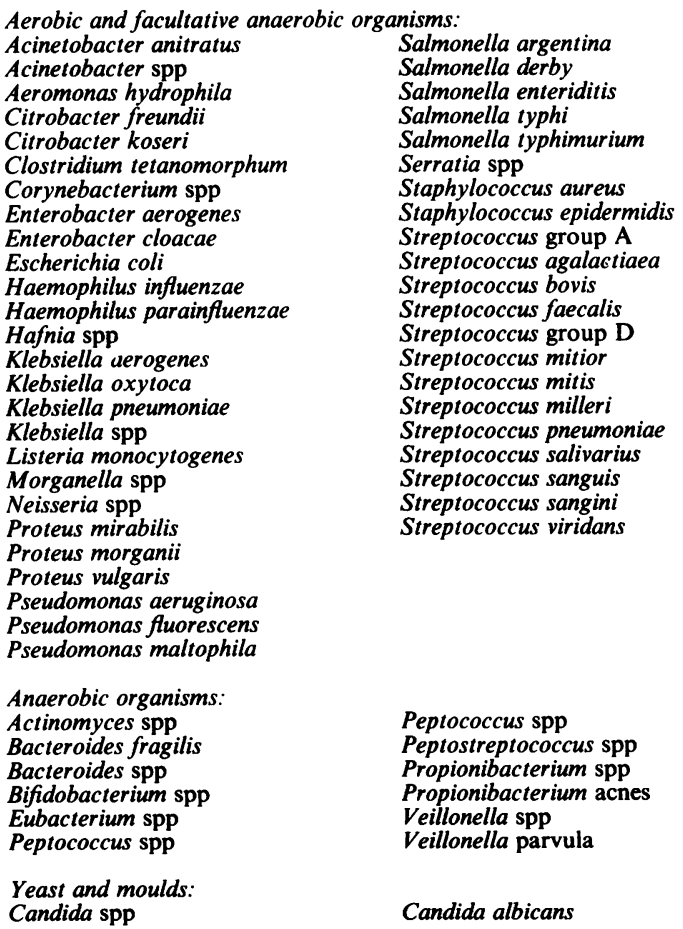

Polymicrobic isolates:

Enterobacter cloacae with Streptococcus viridans

Escherichia coli with Listeria monocytogenes

Escherichia coli with Staphylococcus aureus

Escherichia coli with Streptococcus faecalis

Escherichia coli with Streptococcus viridans

Peptococcus spp with Veillonella spp

Proteus mirabilis with Streptococcus faecalis

Staphylococcus aureus with Streptococcus faecalis
3 Simultaneously $1 \mathrm{ml}$ of each dilution was added to separate blood culture bottles.

4 Freshly collected human blood $(5 \mathrm{ml})$ was added to each bottle.

5 The signal device was inserted aseptically through the rubber diaphragm.

6 The bottles were incubated at $35^{\circ} \mathrm{C}$ and agitated gently twice a day (morning and late afternoon).

7 The detection time for positive signal was recorded (tables 2 and 3 ).

8 At the end of the experiment all the positive blood cultures were subcultured to check the purity and viability of the test organism.

9 The microbial challenge for each test organism was calculated as the number of colony forming units per millilitre on the agar plates.

\section{GAS ANALYSIS OF THE BLOOD CULTURE} BOTTLES HEAD SPACE

Methodology

1 Each bottle was inoculated with $5 \mathrm{ml}$ of freshly collected human blood.

2 Head space gas in one set of bottles was analysed after 15 minutes for percentage carbon dioxide, oxygen, and nitrogen by gas chromatography. The other set of bottles were retained for two hours at $20^{\circ} \mathrm{C}$ and then analysed for carbon dioxide, oxygen, and nitrogen.

3 Further head space gas analyses were made after venting - that is insertion of the signal device and subsequently after two and 24 hours' incubation.

Table 4 shows the results recorded.

Table 5 shows the total range of micro-organisms that were inoculated into the blood culture system and which showed a positive signal after incubation.

Table 6 shows the total range of micro-organisms isolated from blood cultures during clinical trials. The individual trials will be reported separately by the investigators.

\section{Results}

Tables 2, 3, 5 and 6 show the wide range of organisms which grow in the medium, giving positive signals in the upper chamber. Table 4 shows that human blood will give an atmosphere of $5 \%$ carbon doxide in the head space after 24 hours' incubation.

\section{Discussion}

A blood culture system was developed which can detect positive blood cultures by a visual signal. The system overcomes the need for routine subcultures required in conventional methods and therefore reduces effort and laboratory acquired contamination. The two possible sources of laboratory 
contamination with this system are: inadequate sterilisation of the rubber diaphragm prior to attachment of the signal device; and handling of the needle shaft. Experience so far has shown that these sources of contamination can be eliminated with suitable instruction.

The provision of a single medium is an advantage with a small volume of blood and obviates the need to decide which medium to use. The system caters for the capnoeic organisms by providing carbon dioxide in the medium and head space of the bottle. The source of carbon dioxide is the blood and its effect on medium substrates. The venting of the system ensures that the infected blood and broth sample can be obtained at ambient pressure. The simulated human blood culture trial results show that the system is capable of detecting small numbers of organisms in blood samples.

A blood culture system can only be tested in clinical trials and these are under way. The results obtained so far suggest that the principles of the new device are holding in blood culture tests.

The published data from such trials will show that the described system does not exactly match other systems in comparative trials. Although the numbers of clinically important positive isolates are usually similar, examples occur where no growth is seen in the novel system but growth is present in the comparative system. Similarly, examples of the reverse situation will be described.

It is unlikely that any blood culture system is $100 \%$ effective - this novel system included.

In the analysis of positive and negative bottles the numbers and state of the micro-organisms taken from the patient must be considered, together with their distribution in the aliquots of blood injected into the comparative systems. Then the ability of such organisms to grow in the media chosen for the tests becomes a third compounding factor in the analysis.

Confirmation of growth of aerobic, anaerobic, and microaerophilic organisms in the single bottle has been shown with in vitro tests. Again this characteristic must be proved in clinical trials, and such work that has been completed suggests that the bottle does support a wide spectrum of organisms with varying oxygen demands.

The speed at which such organisms are detected, either by visible changes in the broth or by positive signals in the upper chamber, suggests that the novel system operates at about the same speed as the radioactive system. Considerable differences in time scale, however, occur between different genera, species, and strains of organisms.

The system's simplicity is the non-requirement for electronics, hardware, or isotopes; the flexibility is that coping with varying work loads and ease of handling have made this novel system attractive to those workers using it in field trials. The ultimate acceptance of the system will depend on the results of clinical trials currently under way.

We are grateful to Dr P Boswell of Basingstoke District Hospital for his support and organisation of the hospital studies.

Requests for reprints to: Mr EY Bridson, Oxoid Limited, Wade Road, Basingstoke, Hampshire RG24 OPW, England. 